\title{
Guest Editorial: Special Issue on 2011 International Conference on Embedded Computer Systems: Architectures, Modeling and Simulation (SAMOS XI)
}

\author{
John McAllister • Luigi Carro • \\ Skevos Evripidou
}

Received: 15 October 2012 / Accepted: 3 November 2012 / Published online: 20 November 2012 (C) Springer Science+Business Media New York 2012

This special edition of IJPP includes extended versions of the six best papers from the 2011 IEEE International Conference on Embedded Computer Systems: Architectures, Modeling and Simulation (SAMOS XI). The six papers were selected from 37 high quality papers presented at the conference, which had an overall acceptance rate of $41 \%$.

Held in Agios Konstantinos, Samos, Greece from 18th to 21st July 2011, SAMOS (http://www..samos-conference.com) is a unique annual event on embedded computing systems, bringing together researchers from industry and academic in a uniquely relaxing atmosphere which combines dissemination of state-of-the-art research with afternoon beach and boat trips and day-long hikes in the mountains, all bathed in the glorious sunshine of Samos in July. In every sense of the word, SAMOS is a unique event.

The first article included, 'ADL-Based Specification of Implementation Styles for Functional Simulators' by David Penry, and Kurtis Cahill describes a novel orthogonal-specification design principle for use in the development of microarchitectural functional simulators. This approach, which specifies how a simulator is implemented separately from what it is implementing, enables different styles of simulator to be automatically synthesized.

\footnotetext{
J. McAllister $(\varangle)$

Queen's University Belfast, Belfast, UK

e-mail: j.Mcallister@ecit.qub.ac.uk

L. Carro

Universidade Referal Do Rio Grande Do Sul, Porto Alegre, Brazil

S. Evripidou

University of Cyprus, Nicosia, Cyprus
} 
In 'A Parallel Dynamic Binary Translator for Efficient Multi-Core Simulation', Volker Seeker et al., describe a fast, scalable simulation methodology for multi-core platforms based on dynamic binary translation. Their approach has enabled simulation speeds of up to 25,307 MIPs on 32-core $\times 86$ host machines for up to 2,048 processorssimulation performance over two orders of magnitude in advance of leading FPGA architecture simulation technology.

'Scalable Unified Transform Architecture for Advanced Video Coding Embedded Systems' by Tiago Manuel Dias et al. describes a high throughput unified architecture for computing transform function in video codecs which can be used as a hardware accelerator for the 2D $4 \times 4$ and $2 \times 2$ transforms in the H.264/AVC standard. This architecture achieve speedup by a factor of around 120 over pure software implementations of the transform algorithms, allowing real-time transforms for 4,320 $\times 7,680$ Ultra-High Definition Video sequences.

In 'UniTi: Unified Composition and Time for Multi-Domain Model-Based Design', Rovers and Kuper detail a comprehensive description of their multi-domain modelling and simulation environment for continuous-time, discrete-time and dataflow systems, verifying the approach using a phased-array beamforming case study.

Sundararajan, Jones and Topham use their paper entitled 'The Smart Cache: An Energy-Efficient Cache Architecture Through Dynamic Cache Adaptation' to describe their unique approach to cache design for low-power embedded systems design. Their reconfigurable cache architecture demonstrates energy-delay characteristics on average 70 and $12 \%$ better than baseline for two and four-core systems respectively.

Finally, in 'Using SDRAM Memories For High Performance Accesses To TwoDimensional Matrices Without Transpose', Langemeyer, Pirsch and Blume introduce a new address mapping scheme which exploits the burst capability of modern multibank SDRAM architectures to maximise throughput when reading or writing rows or columns of 2D matrices. When applied to 2D-FFT for radar applications, continuous bandwidth utilization of $96-98 \%$ is achieved.

These six papers cover a diverse range of technologies, spanning application modelling, simulation and computing architectures which exemplifies the unique nature of the SAMOS event, and in particular the diversity of SAMOS XI. 